\title{
Clinical retrospective analysis of cases with multiple primary malignant neoplasms
}

\author{
L.L. Xu and K.S. Gu \\ Department of Oncology, First Affiliated Hospital of Anhui Medical University, \\ Hefei, Anhui, China \\ Corresponding author: K.S. Gu \\ E-mail: gukangshengabc@163.com
}

Genet. Mol. Res. 13 (4): 9271-9284 (2014)

Received May 10, 2013

Accepted August 27, 2013

Published March 12, 2014

DOI http://dx.doi.org/10.4238/2014.March.12.19

\begin{abstract}
The etiological factors, clinical characteristics, diagnosis, treatment strategies, and prognosis of multiple primary malignant neoplasms (MPMNs) were investigated in order to improve its diagnosis and therapy. Clinical data of 170 patients with MPMN who were admitted to the Oncology Department of the First Affiliated Hospital of Anhui Medical University from January 2004 to April 2010 were collected and analyzed retrospectively. The overall incidence of MPMNs was $2.25 \%$. There were 167 cases with duplex primary cancers, 2 cases with triple primary cancers, 1 case with quadruple primary cancer, 46 synchronous MPMNs (SMPMNs), and 124 metachronous MPMNs (MMPMNs). There were 344 malignant neoplasms in the 170 MPMN patients, of which 161 were in the alimentary system, 48 in the respiratory system, 63 in mammary glands, 30 in the genital system, 7 in the urinary system, 23 in the head and neck, and the other 12 were in the brain, hematological system, and soft tissue. There were 22 cases of bilateral primary breast cancer and 41 cases of digestive MPMN. With a median survival time of 24 months, the 1-, 3-, and 5 -year cumulative survival rates in the 170 patients were $68.8,39.1$, and $25.2 \%$, respectively. The most common locations of MPMN were the alimentary system, mammary gland, and respiratory system. MPMN
\end{abstract}


usually occurred in distinct organs of identical systems, conjugate organs, or identical organs. The survival rate of SMPMN was similar to that of MMPMN. There were differences in principles of treatment and diagnosis between MPMN and metastasis or recurrent cancers.

Key words: Multiple primary malignant neoplasms (MPMN); Neoplasms; Synchronous MPMN; Metachronous MPMN

\section{INTRODUCTION}

Multiple primary malignant neoplasms (MPMNs) are defined as two or more unrelated primary malignant tumors that originate from different organs and occur in the body at the same time or one after another. According to the tumor discovery time interval, MPMNs can be divided into two main categories: synchronous MPMNs (SMPMNs) and metachronous MPMNs (MMPMNs). SMPMNs are defined if the tumors occur simultaneously or within 6 months of one another, whereas MMPMNs are indicated if the interval time is more than 6 months. The incidence of MMPMN is lower than SMPMN.

Clinically, MPMN is often confused with metastasis or recurrence of malignant tumors since both are characterized by new lesions (Cigna et al., 2011). Metastatic tumors are derived from the primary lesion, with both showing the same pathological characteristics and similar developmental processes and prognosis. Conversely, MPMN refers to the development of a new malignant lesion de novo. In MPMN, the characteristics of the new lesions are completely different from those of the original tumor lesions (Papajík et al., 2011). Therefore, the prognosis, metastasis, and recurrence are also completely different between MPMN and the metastasis or recurrence of malignant tumors.

The occurrence of MPMN has increased in recent years. Because of its great relevance to tumorigenesis, MPMN patients in our hospital were investigated to explore the clinical characteristics, diagnosis, treatment principles, and prognosis of MPMN in order to improve its diagnosis and therapy and to prolong survival.

\section{MATERIAL AND METHODS}

\section{Diagnostic criteria}

The diagnostic principles of MPMN are based on the following standards provided by Warren (Warren and Gates, 1932), combined with the Liu Fusheng's added standard (Liu et al., 1979): 1) each tumor is malignant, 2) each tumor has its own pathological features, 3) tumors occur in different parts or organs, and are not continuous with each other, and 4) each tumor has its own metastatic pathway and the diagnosis of metastatic or recurrent tumors can be excluded.

The following diagnostic criteria of bilateral primary breast cancer (BPBC) were used: 1) primary carcinomas are found in bilateral breast tissue, e.g., ductal carcinoma or lobular carcinoma, 2) the histopathological types of breast cancer are different on each side, 3) bilateral breast cancer shows the same histopathological types along with no local recurrence, lymphatic metastasis, or other distant metastases, 4) primary cancer often occurs at the outer 
upper quadrant as well as in the breast parenchyma, is ill-defined and often infiltrates, whereas metastatic cancer is more common inside of the contralateral breast or tail adipose tissue, with the expansion of growth, and is often multiple, and 5) the breast cancer occurs more than 5 years after treatment of the first primary breast cancer, and is contralateral to the occurrence of the first primary breast cancer. According to the time interval of 6 months, BPBC was divided into synchronous BPBC (SBPBC) and metachronous BPBC (MBPBC) $(\mathrm{He}, 2010)$.

\section{Data analysis}

We retrospectively analyzed 7547 cases with malignant tumors in patients admitted to the First Affiliated Hospital of Anhui Medical University between January 2004 and April 2010. In line with the diagnostic criteria described above, 170 patients who had complete follow-up data were diagnosed as MPMN, representing an overall rate of $2.25 \%$. Data collection included gender, age, medical history, personal history, family history, treatment, and primary tumor histological type, location, and time of onset. Ninety cases were males and 80 cases were females. Forty-six patients were diagnosed as SMPMN (31 males, 15 females), and 124 cases were diagnosed as MMPMN (59 males, 65 females). The median age of MMPMN was 60 years old (range, 32 to 77 years); 10 cases were under and including 50 years old, and 36 cases were older than 50 years old. Patients with MMPMN had occurrence of the first tumor at a median age of 56 years (range, 30 to 86 years), and were diagnosed with the second tumor at a median age of 61 years (range, 34 to 90 years); 28 cases were less than 50 years old and 96 cases were more than 50 years old. A total of 19 patients had a family history of malignant tumors, accounting for $11.2 \%$.

\section{Follow-up}

Overall survival and median survival were used as indicators to assess survival time. Overall survival was calculated from the tumor diagnosis date to the death or last follow-up date; if the patient was lost, the last follow-up date was defined as the study endpoint.

For patients with SMPMN, the survival time was calculated according to the confirmed date of the first tumor, whereas for patients with MMPMN, survival time was calculated from the confirmed date of the last diagnosis of the tumor.

A total of 170 patients were followed up with the deadline of February 1, 2011 by either telephone or in-patient. Six cases were lost, and the follow-up rate was $96.5 \%$; 50 cases remain alive to date.

\section{Statistical analysis}

All data were statistically analyzed using the SPSS16.0 software; the median was used for count data, and the chi-squared test was used to evaluate differences in the location in SMPMN and MMPMN. We calculated the median survival time by the Kaplan-Meier method to obtain survival curves. The log-rank test was used to compare the survival time between different groups, and $\mathrm{P}<0.05$ was considered to be statistically significant. Statistical significance of observed differences between groups were determined by the Student $t$-test and one-way analysis of variance (ANOVA). Values of $\mathrm{P}<0.05$ were considered to be significant 


\section{RESULTS}

\section{Tumor distribution}

Of the total 170 MPMN patients, 167 cases were duplex primary malignant neoplasms (DPMN), 2 cases were triple primary malignant neoplasms (TPMN), and 1 case was quadruple primary malignant neoplasm (QPMN). DPMN was the most common with a total of 344 primary tumor lesions (Table 1), including two cases of primary liver cancer combined with a history of hepatitis, which were clinically diagnosed using alpha-fetoprotein radiological methods combined with family history; other diagnoses were confirmed by pathological or cytological analyses.

Table 1. Distribution of tumor.
\begin{tabular}{lrrrrrrrrrrrrrr}
\hline Classification & SM & ESO & LI & SI & HP & G & PAN & L & BR & U & RP & HN & Others & Total \\
\hline First tumor & 21 & 9 & 22 & 3 & 0 & 2 & 0 & 7 & 28 & 13 & 4 & 12 & 3 & 124 \\
Second tumor & 12 & 11 & 16 & 2 & 3 & 0 & 1 & 33 & 18 & 11 & 2 & 8 & 7 & 124 \\
Third tumor & 1 & 0 & 1 & 0 & 0 & 0 & 0 & 0 & 1 & 0 & 0 & 0 & 0 & 3 \\
Fourth tumor & 1 & 0 & 0 & 0 & 0 & 0 & 0 & 0 & 0 & 0 & 0 & 0 & 0 & 1 \\
Simultaneous & 27 & 14 & 9 & 1 & 3 & 2 & 0 & 8 & 16 & 6 & 1 & 3 & 2 & 92 \\
Total & 62 & 34 & 48 & 6 & 6 & 4 & 1 & 48 & 63 & 30 & 7 & 23 & 12 & 344 \\
\hline
\end{tabular}

$\mathrm{SM}=$ stomach ESO = esophagus; $\mathrm{LI}=$ large intestine; $\mathrm{SI}=$ small intestine; $\mathrm{HP}=$ hepatobiliary; $\mathrm{G}=$ gallbladder; $\mathrm{PAN}=$ pancreas $; \mathrm{L}=$ lung; $\mathrm{BR}=$ breast $\mathrm{U}=$ urinary tract $\mathrm{RP}=$ reproductive system; $\mathrm{HN}=$ head and neck.

A total of 161 of the 344 tumor lesions were located in the digestive system, 48 in the respiratory system, 63 in the breast, 7 in the reproductive system, 30 in the urinary system, 23 in the head and neck, and the other 12 occurred in the brain, blood system, and soft tissue.

Ten cases of the 46 SMPMN patients' tumors occurred in different organs of different systems, 22 cases occurred in different organs of the same system, 8 cases occurred in paired organs, and 6 cases occurred in the same organs. One hundred and twenty-four cases of MMPMN and the one case of QPMN occurred in different organs or the same organs of the same system, 83 cases occurred in different organs of different systems, 19 cases occurred in different organs of the same system, 16 cases occurred in paired organs, and 5 cases occurred in the same organ.

The incidence of primary tumors in different organs of different systems was higher in MMPMN than in SMPMN, whereas the incidence of primary tumors occurring in different organs or in the same organ of the same system was significantly higher in SMPMN than in MMPMN $(\mathrm{P}<0.05)$. There was no significant difference between MMPMN and SMPMN in the incidence of tumors occurring in paired organs (Table 2).

According to the common tumor pathological classification of the World Health Organization, there were 344 primary tumor lesions, 318 of which originated from the epithelial tissue, 4 from a source of mesenchymal tissue, 16 from the hematopoietic and lymphoid tissues, and 6 from other sources, including nerve tissue, germ cells, and placental trophoblast cells (Table 3). 
Table 2. Distribution of primary tumors in SMPMN and MMPMN.

\begin{tabular}{lcccc}
\hline & SMPMN & MMPMN & $\chi^{2}$ & P value \\
\hline DSDO & 10 & 83 & 28.305 & $<0.001$ \\
SSDO & 22 & 19 & 19.102 & $<0.001$ \\
PO & 8 & 16 & 0.528 & 0.309 \\
SO & 6 & 5 & 4.435 & 0.045 \\
\hline
\end{tabular}

$\mathrm{DSDO}=$ different system and different organ; SSDO = same system and different organ; PO = paired organ; SO $=$ same organ.

Table 3. Distribution of primary tumors based on pathological classification.

\begin{tabular}{lcccc}
\hline & Epithelial & Mesenchymal & Hematopoietic/lymphoid & Others \\
\hline First tumor & 114 & 0 & 5 & 5 \\
Second tumor & 112 & 2 & 0 & 0 \\
Third tumor & 3 & 0 & 0 & 0 \\
Fourth tumor & 1 & 2 & 1 & 0 \\
Simultaneous & 88 & 4 & 16 & 6 \\
Total & 318 & &
\end{tabular}

\section{Tumor interval time}

The tumor interval time is defined as twice the diagnosis of cancer, including endoscopic, surgical pathology, and cytological tests for diagnoses of the cancer interval time. In 124 cases with MMPMN, the median interval time between the first and second tumor was 41.5 months (range, 7-40.8 months), in 13 cases, the interval time was $<1$ year, in 15 cases it was 1 to 2 years, in 18 cases it was 2 to 3 years, in 11 cases it was 3 to 4 years, in 12 cases it was 4 to 5 years, and in 46 cases it was more than 5 years.

In the two TPMN patients, the interval times between the second and third tumor were 5 and 16 months, respectively. In the QPMN case, the interval time between the second and third tumor was 204 months and that between the third and fourth tumor was 28 months.

Tumors of 46 cases of SMPMN were diagnosed by surgery or endoscopy at the same time, and the interval time of the other 6 cases ranged from 2 to 6 months.

\section{Tumor treatment}

All of the 344 primary tumors including digestive system cancer, lung cancer, breast cancer, gynecological system cancer, and urinary system cancer were treated by operation and patients received chemotherapy or radiotherapy after surgery, depending on the circumstances. Primary liver cancer was mainly treated with transcatheter arterial chemoembolization (TACE), head and neck cancer was treated with radiotherapy with partial combination of chemotherapy, blood system tumors were treated with chemotherapy partially combined with radiotherapy, and hormone-dependent tumors, such as prostate cancer and both estrogen receptor- and progesterone receptor-positive breast cancer, were treated with endocrine therapy.

The treatments for primary tumor foci are shown in Table 4. Eighty-three cases of primary tumors underwent surgical resection. Of the 124 MMPMN patients with primary tumor foci, 111 cases $(89.5 \%)$ underwent surgery, 77 cases $(62.1 \%)$ were treated with chemotherapy, and 21 cases $(16.9 \%)$ were treated with radiotherapy. Of the 124 second primary tumor foci, 
surgery was performed in 56 cases (45.2\%), chemotherapy was administered in 102 cases $(82.3 \%)$, and radiotherapy was administered in 23 cases $(18.5 \%)$. In the total of 92 primary tumor lesions of SMPMN, there were 59 cases of surgery (64.1\%), 82 cases of chemotherapy (89.1\%), and 11 cases of radiotherapy $(12.0 \%)$.

\begin{tabular}{|c|c|c|c|c|c|}
\hline & Surgery & Chemotherapy & Radiotherapy & Endocrine treatment & TACE \\
\hline First tumor & 111 & 77 & 21 & 9 & 0 \\
\hline Second tumor & 56 & 102 & 23 & 6 & 3 \\
\hline Third tumor & 2 & 2 & 0 & 1 & 0 \\
\hline Fourth tumor & 1 & 0 & 0 & 0 & 0 \\
\hline Simultaneous & 59 & 82 & 11 & 6 & 1 \\
\hline
\end{tabular}

$\mathrm{TACE}=$ transcatheter arterial chemoembolization

\section{Survival and prognostic factors}

A total of 170 patients were followed up between 3 to 434 months, with a median follow-up time of 52.5 months. The median survival of all patients was 24 months $(95 \%$ confidence interval $(\mathrm{CI})=16.9-31.1)$, and the total 1-, 3-, and 5-year survival rates were 68.8, 39.1, and $25.2 \%$, respectively. The overall survival curve is shown in Figure 1.

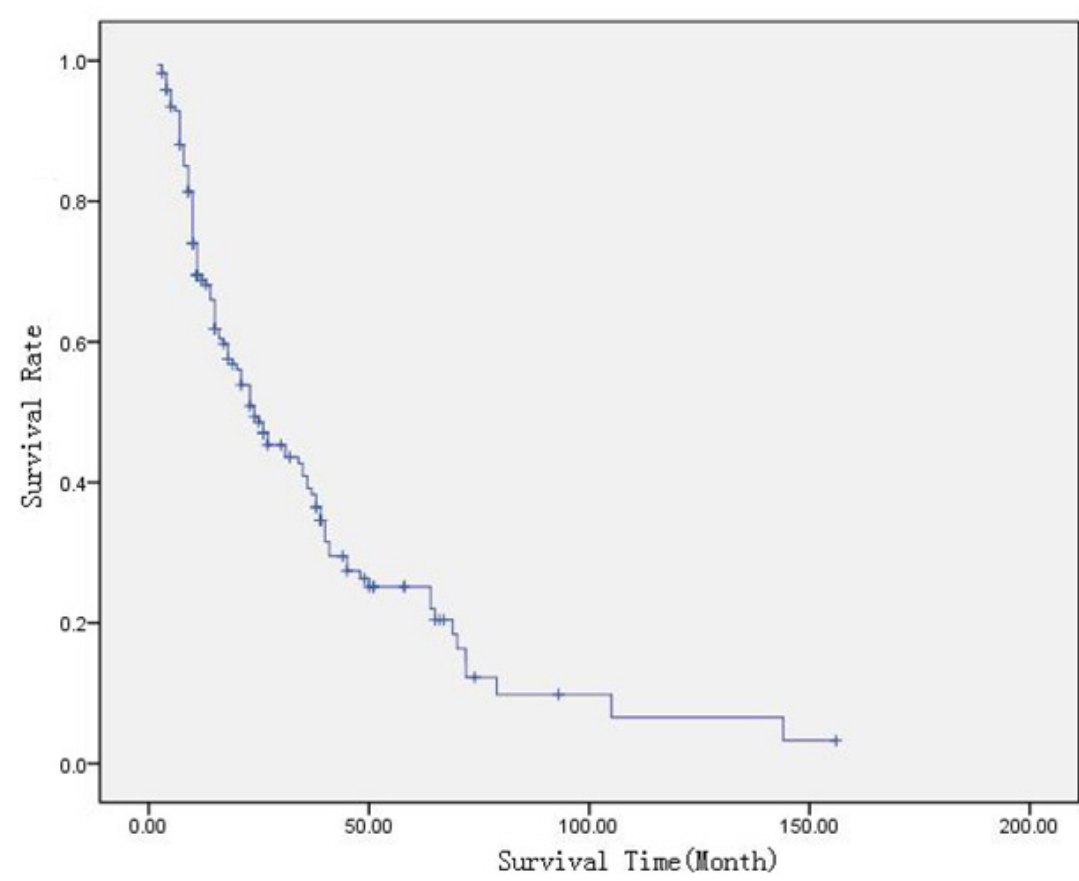

- TSurvival Function + Censored

Figure 1. Kaplan-Meier survival curve of 170 patients with MPMN.

The median survival of the first tumor in the 121 DPMN patients was 90 months $(95 \% \mathrm{CI}=66.8-113.2$ months), and the 5- and 10-year survival rates were 66.4 and $42.1 \%$, re- 
spectively. The median survival time of the second tumor was 25 months $(95 \% \mathrm{CI}=17.2-32.8$ months), and the 3- and 5-year survival rates were 37.8 and $25.1 \%$, respectively. The median survival of the 46 SMPMN patients was 21 months ( $95 \% \mathrm{CI}=0-50.4$ months), and the 3 - and 5 -year survival rates were 48.3 and $26.8 \%$, respectively.

\section{Influence of interval time on survival}

There was no significant difference in survival time between SMPMN and MMPMN $(\mathrm{P}=0.81)$, as shown in Figure 2.

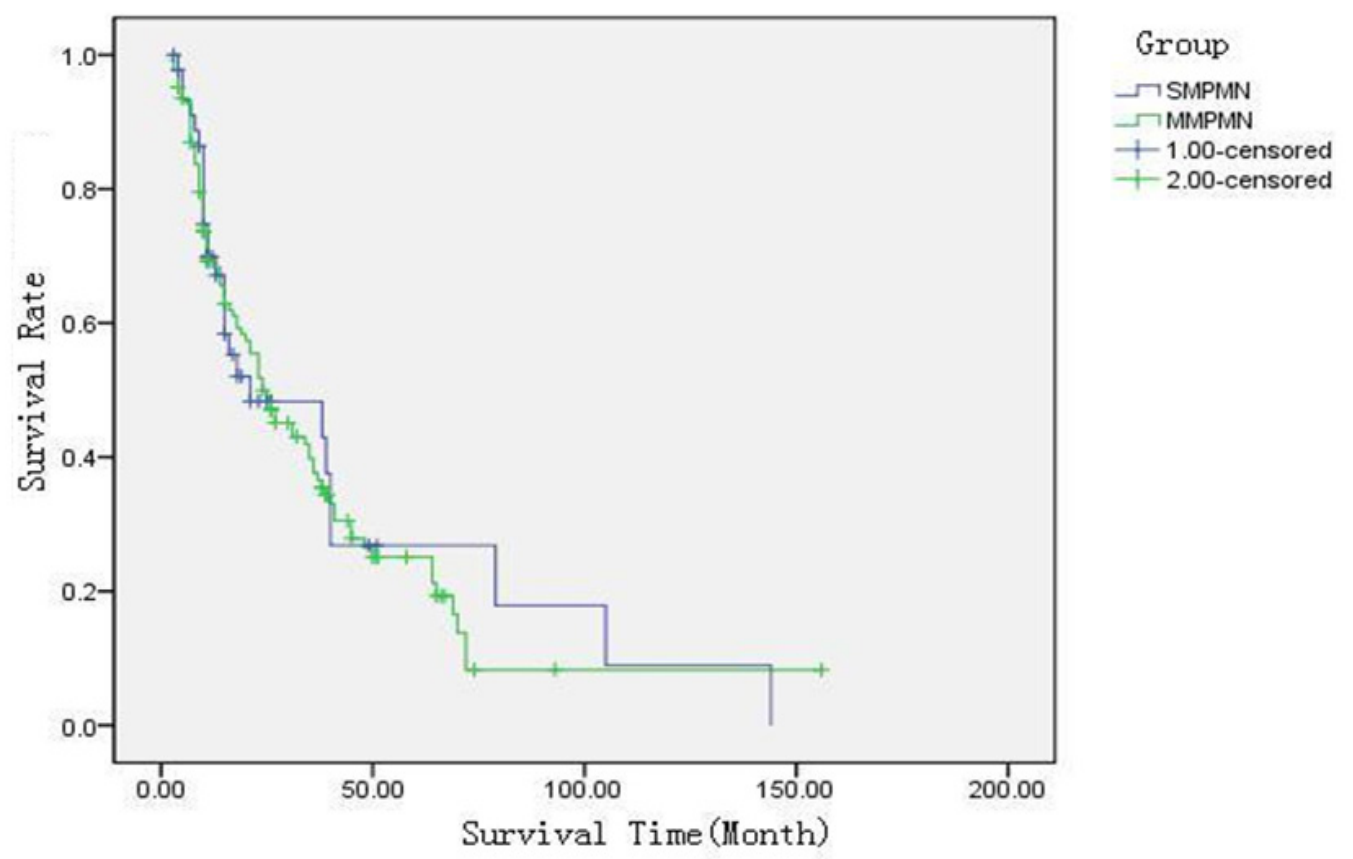

Figure 2. Comparison of patient proportion survival rate between SMPMN and MMPMN.

According to the interval time of 5 years, 167 cases of DPMN were divided into two groups: 46 cases were greater than 5 years, and 121 cases, including SMPMN, were less than or equal to 5 years. The survival rate was lower in the group with an interval time of less than 5 years compared to the group of 5 years or more (Figure 3 ), but the difference was not statistically significant $(\mathrm{P}=0.558)$.

\section{Surgical treatment influence on survival}

The 170 patients were divided into two groups according to the surgical treatment of primary tumors: 83 cases (29 cases of SMPMN and 54 cases of MMPMN) underwent surgical 
resection and 87 cases did not receive complete surgical resection (60 cases of partial surgery and 27 cases of no surgery).

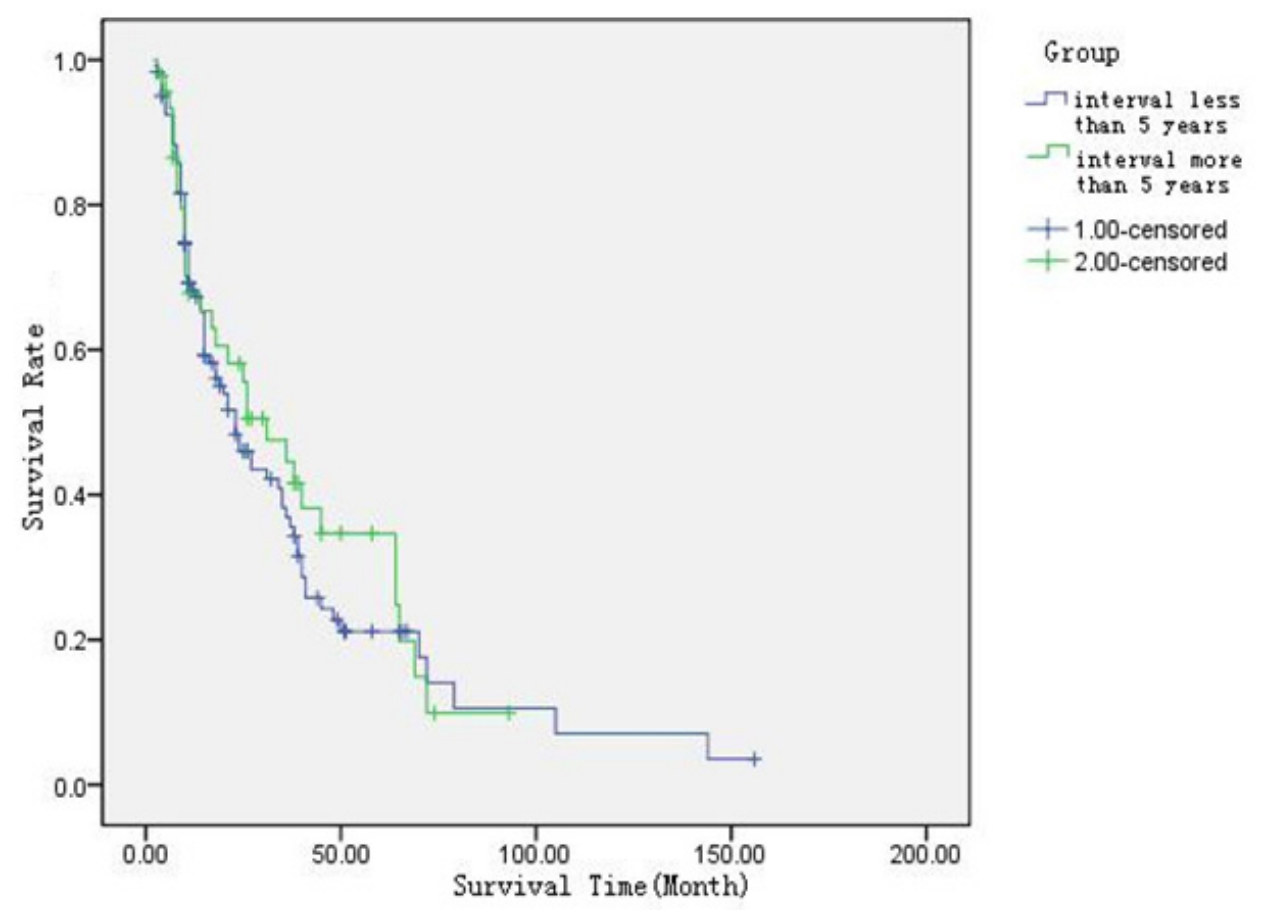

Figure 3. Comparison of patient proportion survival rate between occurrence interval less and more than 5 years.

The median survival of the primary tumor surgery group was 45 months $(95 \% \mathrm{CI}=$ 35.5-54.5 months) and that of the group in which primary tumors were not operated was 11 months $(95 \% \mathrm{CI}=8.1-13.9$ months $)$, and the difference was statistically significant $(\mathrm{P}<0.001)$ as shown in Figure 4.

\section{Specific MPMNs}

\section{$B P B C$}

Based on diagnostic criteria, a total of 22 cases were diagnosed as $\mathrm{BPBC}$, including 7 cases of SBPBC and 15 cases of MBPBC. The shortest interval time was 12 months, with a median interval time of 39 months. Ten cases had time intervals $<5$ years; 5 cases were greater than 5 years (219 months) and 2 cases were greater than 10 years (228 months).

The 22 BPBC patients were diagnosed at a median age of 49 years (range, 36 to 72 years). Bilateral breast lesions surgery was performed in 20 cases, and in two cases surgery was performed on the first side of the lesion but not on the second side of the lesion.

The median survival time of the $22 \mathrm{BPBC}$ patients was 39 months $(95 \% \mathrm{CI}=36.5$ 41.5 months), the 3 -year survival rate was $81.1 \%$, and the 5 -year survival rate was $37.4 \%$. 


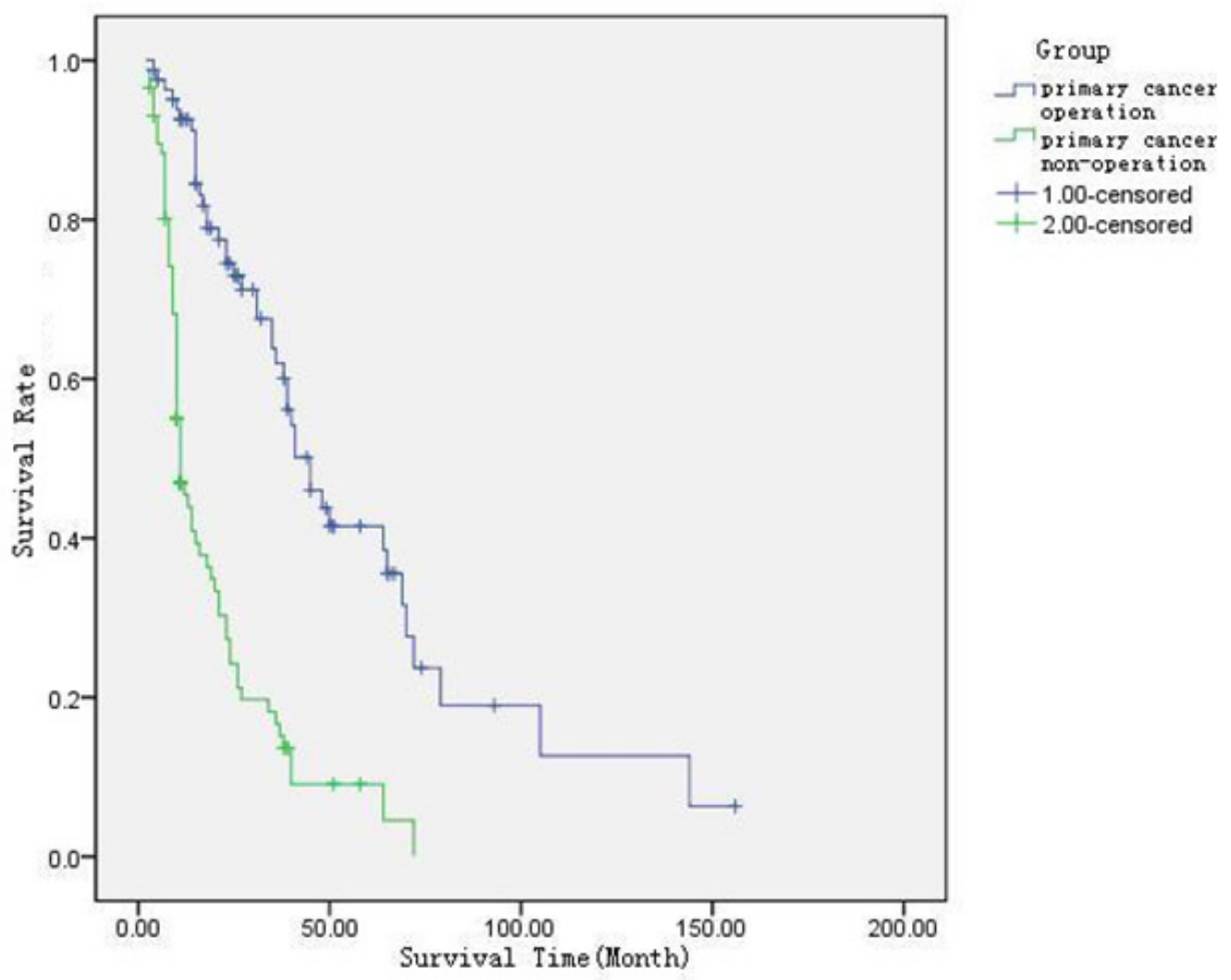

Figure 4. Comparison of patient proportion survival rate in different radical operation.

\section{Digestive tract multiple primary malignant neoplasms}

Digestive tract MPMNs refer to primary malignant tumors at various places of the tract including the esophagus, stomach, small intestine, and large intestine.

There were 41 cases of gastrointestinal MPMN, except for 1 case of gastrointestinal TPMN, 17 cases of stomach, esophagus DPMN (12 cases of SMPMN and 5 cases of MMPMN), 12 cases of stomach, intestinal DPMN (2 cases of SMPMN and 10 cases of MMPMN), 7 cases in the gut ( 2 cases of SMPMN and 5 cases of MMPMN), and 4 cases of gastric multiple primary malignant tumors (both SMPMN); 19 cases of paired tumors were surgically resected.

Of the 20 cases of gastrointestinal SMPMN, eight cases had complete surgery, 10 cases had incomplete surgery, and 2 cases had no surgery. Of the 20 cases of gastrointestinal MMPMN, 1 case had complete surgery and 19 cases had incomplete surgery.

The median survival time of the 42 patients was 40 months $(95 \% \mathrm{CI}=32.9$ 77.1 months), the 3 -year survival rate was $57.7 \%$, and the 5 -year survival rate was $35.1 \%$. 


\section{DISCUSSION}

In patients with cancer, the incidence of new tumor generation is approximately 1 to $10 \%$, which is much higher than that of the normal population. In this study, the occurrence rate of MPMN was $2.25 \%$, which is the same as previously reported. MMPMN was more frequent than SMPMN, with a ratio of 2.7:1; second primary tumors were most common, whereas third, fourth, and higher primary tumors were relatively rare. In addition, the incidence of MPMN increased with age (Demandante et al., 2003). As reported previously, the median age of second primary tumor cases was 56.4 years, and the age group of more than 50 years old accounted for $79.4 \%(\mathrm{~N}=63)$ of such cases. The age of onset did not differ significantly between SMPMN and MMPMN, whereas the occurrence of second tumor and age were significantly correlated $(\mathrm{r}=0.99)$ (Irimie et al., 2010).

The predilection site of MPMNs varies greatly according to the tumor distribution of various regions and the different patients admitted. In Japan, MPMN of gastrointestinal tumors are the most common, the majority of which are gastric cancer. In the Guangdong region of China, nasopharyngeal cancer is the most common MPMN. In the present study, the original tumor sites were most commonly observed in the digestive system, followed by the breast, respiratory system, reproductive system, and head and neck. BPBC and gastrointestinal primary malignant tumors were found the most frequently because patients with digestive system cancer and breast cancer are more frequently admitted to our hospital. Previous studies have reported that the most common first primary tumor organ is the uterus, followed by the breast, esophagus, and lung, whereas the most common second primary cancer tumor site is the lung, followed by the esophagus, breast, uterus, and rectum. In this study, the first tumor occurred most frequently in the breast, followed by the colon, stomach, esophagus, and lung, whereas the second tumor occurred most frequently in the lung, followed by the breast, colon, stomach, and esophagus. Simultaneous tumors were most frequently found in the stomach, followed by the breast, esophagus, colon, and lung. MPMN occurred most commonly in the same organ in one system, in paired organs, or in the same organ, accounting for $45.3 \%$ of all cases. The incidence of SMPMN in the same organs in different systems was higher than that of MMPMN. The most common type of second primary tumor was skin cancer, followed by breast cancer, acute leukemia, colorectal cancer, lung cancer, and stomach cancer, including two categories: solid tumors and lymphoid hematopoietic system tumors. The ratio between the two tumor types observed here differed from that reported previously (Morgenfeld et al., 2003), which might be related to differences in treatment, region, race, disease, and/or statistical methods. A follow-up study of chemotherapy alone or the combined treatment of chemotherapy with radiotherapy for Hodgkin's lymphoma (HL) found that the combined treatment more commonly resulted in leukemia than did radiotherapy alone. Radiation-induced second primary cancer usually occurs within radiotherapy wild, or radiotherapy wild around, and solid tumors are more common (Aleman et al., 2003). Our results showed that multiple primary tumors derived from the hematopoietic and lymphoid system were rare, while solid tumors represented the vast majority and were originally from epithelial tissues, which may be associated with the tumor incidence of this region, treatment, and received treatment species, among other factors.

The cause of MPMN is not yet elucidated, but may be related to the following four main factors. First, intrinsic factors can lead to MPMN, which include susceptibility, immune status, and endocrine and embryonic development. Immune surveillance and immune defenses 
will not function in cancer patients, and cancer-causing factors will have increased sensitivity to those prone to MPMN (Yamamoto et al., 2006).

The second cause is related to environmental factors and personal lifestyle, including long-term exposure to radiation and industrial pollution of the environment. If the mucosal epithelium of the head and neck, lung, or esophagus is exposed to the same environmental carcinogen, it will be increasingly susceptible to MPMN. In addition, smoking and alcohol consumption are important carcinogenic factors. Studies have shown that smoking is not only related to the development of small cell lung cancer (SCLC), but that smoking during SCLC treatment can also affect survival.

Third, genetic factors can affect the development of MPMN. Molecular genetic research has provided much evidence of the hereditary nature of tumors, including the genetic susceptibility to hereditary tumors and tumor genetic susceptibility. Hereditary nonpolyposis colorectal cancer (HNPCC) accounts for approximately 0.3 to $5.8 \%$ of colorectal cancers. HNPCC is caused by a variety of genetic factors including mismatch of repair genes and gene mutations, and colorectal cancer is often accompanied by other types of malignant tumors, such as endometrial cancer, ovarian cancer, gastric cancer, liver cancer, and pancreatic cancer (de la Chapelle, 2005). In addition, patients with breast and ovarian cancer commonly show a dominant family history, and this mode of inheritance is autosomal dominant gene mutations (Chaturvedi et al., 2007). Of the 170 MPMN patients evaluated in the present study, only 19 cases $(11.2 \%)$ had a family history of cancer.

The final cause of MPMN is related to therapeutic factors, including carcinogenic radiation therapy and chemotherapy. The carcinogenic factors of radiation therapy-induced second primary cancer have been recognized, which may be due to oncogene activation and DNA damage (Begg et al., 2011). Development of a second primary cancer has been considered to be the most serious remote complication after HL radiotherapy, and is one of the leading causes of death in HL, so that the overall survival benefit of radiation therapy was partially offset by the incidence of radiation therapy-induced second primary cancer (Aleman et al., 2003). Anticancer drugs themselves are carcinogenic, and this problem has attracted universal attention. Many anticancer drugs, especially alkylating agents themselves, and chemotherapeutic agents are carcinogens, but the specific carcinogenic mechanism underlying their effects is not yet clear. The mechanism might be associated with chemotherapy drug-induced DNA-protein crosslinkages and/or DNA strand breaks and cell transformations, mutations, and chromosomal aberrations (Noronha et al., 2006). Patients with malignant tumors generally receive long-term chemicals for anticancer treatment and high-dose hormone therapy, resulting in decreased immune function, thus making them more prone to cancer (Rondeau et al., 2010). Immune reconstitution after systemic chemotherapy is not only important for the prevention of cancer recurrence and metastasis, but is also an important measure for the prevention of the occurrence of multiple primary cancers. In this study group, one case of breast cancer that received chest wall radiotherapy for 17 months had two rounds of chest wall pleomorphic leiomyosarcoma in the regions of the original chest wall that the radiotherapy targeted. Two breast cancer patients who were treated with tamoxifen endocrine therapy for 25 and 43 months respectively had two rounds of endometrial cancer.

The essence for establishing MPMN diagnostic criteria is to avoid misdiagnosis and to distinguish between recurrence and metastasis. Early detection and intervention results in much better prognosis compared to single primary malignant recurrence or metastasis. The 
early diagnosis of MMPMN requires strengthened follow-up after the first primary tumor radical treatment, as the immune ability of cancer patients will be compromised, thus greatly increasing the chance of MMPMN. More importantly, MMPMN should not be considered as the first primary malignant tumor recurrence or transfer, and give up the chance of surgery or radical treatment. If new lesions appear in the follow-up study, the specificity of each case should be consulted in detail, with particular respect to the general mechanism of the metastasis of malignant tumors, cellular pathology results should be examined, and importantly, the new lesions should not simply be considered as recurrence of malignant tumors or metastasis.

Recurrence is of great importance for discriminating between MPMN and tumor metastasis because significant differences in treatment principles exist between the two. In metastasis, recurrence of the tumor of the primary cancer has generally progressed to the late stage, the extent of the disease is often not limited, and radical surgery is generally impossible. Therefore, treatment plans are generally developed according to the primary cancer characteristics, biological characteristics, and developmental outcomes, and are mainly focused on palliative therapy rather than on healing (Kim et al., 2008). On the other hand, the possibility to cure second primary cancers is higher, and treatment options should be based on the same principles as used for the assessment of single tumors, i.e., according to the histological type, site, clinical stage, and the patient's physical condition, and treatments should be based on surgery in combination with other treatments including chemotherapy, radiotherapy, and biological methods (Hujala et al., 2005).

There is currently no consensus for the method of calculating the survival rate of MPMN. Many researchers recommend basing the rate from the final diagnosis of the malignancy tumor, while others suggest calculating survival from the diagnosis of the first tumor in order to take into account the increased risk of malignancy tumor occurrence during the prolonged survival period. We recommend using the last cancer diagnosis to calculate the survival rate for determining the prognosis of MPMN. Because MMPMN has a relatively longer interval, which can possibly result in a bias, it is suggested that the calculation of survival time that is based on the last tumor diagnosis is more reasonable. In a study of the survival time of MPMN, Wang et al. (2005) compared second primary SCLC to first primary SCLC and found no significant differences in efficacy and median survival time. Bhattacharyya and Nayak (2005) found that second primary cancer survival rates were higher than those of the first primary tumors of head and neck cancer, but the results were not statistically significant. In addition, the survival rate of second primary cancer was associated with lesion sites.

Although there is not yet a consensus of prognostic factors of MPMN, several studies have shown consistent prognostic factors, including: the MPMN occurrence interval, earlier or later stage of the disease and tumor type, biological characteristics, and whether or not diagnosis and correct treatment are applied in a timely manner, including radical surgery, adjuvant chemotherapy, and radiotherapy.

The definition of SMPMN and MMPMN is the starting point to achieve accurate comparisons, and 6 months is the standard criterion used in most previous studies. According to the biological characteristics of tumors, malignant tumors must be present for a certain period of time before showing clinical manifestations; therefore, if the interval time between tumors is too short, MMPMN might be equivalent to SMPMN, so that the designation of simultaneous or metachronous is merely relative.

In part of the study, the MMPMN interval was set to be greater than 1 year. Survival 
rate comparisons for SMPMN and MMPMN show inconsistent results. Ayhan et al. (2003) found similar survival rates and prognoses between simultaneous and metachronous ovarian and endometrial cancers. Similarly, Oya et al. (2003) found no statistically significant difference in the overall survival rates of simultaneous and metachronous colorectal cancer. In contrast, Zhu et al. (2010) found median survival rates of 1.9 and 4.3 years for SMPMN and MMPMN patients, respectively, which was a statistically significant difference. Carmichael et al. (2002) found that the SBPBC survival rate was lower compared with those of MBPBC and unilateral breast cancer, and SBPBC was also associated with a higher risk of distant metastasis.

In this study, no significant difference was found in the survival rate between SMPMN and MMPMN. Patients with an interval time of 5 years or less had a lower survival rate compared to those with an interval time of more than 5 years, although the difference was not statistically significant. The 1-, 3-, and 5-year survival rates of MPMN patients in this study were $68.8,39.1$, and $25.2 \%$, respectively. In the $22 \mathrm{BPBC}$ patients, the 3 -year survival rate was $81.1 \%$ and the 5 -year survival rate was $37.4 \%$. In the 42 cases of gastrointestinal MPMN, the 3 -year survival rate was $57.7 \%$ and the 5 -year survival rate was $35.1 \%$. The prognoses of BPBC and gastrointestinal MPMN were better than those of other MPMNs. In addition, the primary cancer surgery group had a significantly higher survival rate than that of the incomplete surgical group, indicating that surgery should be the main MPMN treatment method.

In summary, sufficient clinical attention should be paid to MPMN to avoid misdiagnosis and missed diagnosis, and the difference between metastasis and recurrence of carcinomas and MPMN should be established. In addition, early detection, early diagnosis, and radical treatment are all essential for efficient treatment of MPMN. The stage of disease and site selection should be used to determine surgically based treatment options, combined with chemotherapy, radiotherapy, biological therapy, and other methods to improve survival.

\section{REFERENCES}

Aleman BM, van den Belt-Dusebout AW, Klokman WJ, Van't Veer MB, et al. (2003). Long-term cause-specific mortality of patients treated for Hodgkin's disease. J. Clin. Oncol. 21: 3431-3439.

Ayhan A, Guvenal T, Coskun F, Basaran M, et al. (2003). Survival and prognostic factors in patients with synchronous ovarian and endometrial cancers and endometrial cancers metastatic to the ovaries. Eur. J. Gynaecol. Oncol. 24: 171-174.

Begg AC, Stewart FA and Vens C (2011). Strategies to improve radiotherapy with targeted drugs. Nat. Rev. Cancer 11: 239-253.

Bhattacharyya N and Nayak VK (2005). Survival outcomes for second primary head and neck cancer: a matched analysis. Otolaryngol. Head Neck Surg. 132: 63-68.

Carmichael AR, Bendall S, Lockerbie L, Prescott R, et al. (2002). The long-term outcome of synchronous bilateral breast cancer is worse than metachronous or unilateral tumours. Eur. J. Surg. Oncol. 28: 388-391.

Chaturvedi AK, Engels EA, Gilbert ES, Chen BE, et al. (2007). Second cancers among 104,760 survivors of cervical cancer: evaluation of long-term risk. J. Natl. Cancer Inst. 99: 1634-1643.

Cigna E, Gradilone A, Sorvillo V and Scuderi N (2011). ABCB5 in peripheral blood of a patient affected by multiple primary malignancies. Ann. Ital. Chir. 82: 49-53.

de la Chapelle A (2005). The incidence of Lynch syndrome. Fam. Cancer 4: 233-237.

Demandante CG, Troyer DA and Miles TP (2003). Multiple primary malignant neoplasms: case report and a comprehensive review of the literature. Am. J. Clin. Oncol. 26: 79-83.

He JJ (2010). Bilateral primary breast cancer: an analysis of 2942 cases. China Mod. Doctor 48: 8-10.

Hujala K, Sipila J and Grenman R (2005). Panendoscopy and synchronous second primary tumors in head and neck cancer patients. Eur. Arch. Otorhinolaryngol. 262: 17-20.

Irimie A, Achimas-Cadariu P, Burz C and Puscas E (2010). Multiple primary malignancies - epidemiological analysis at a single tertiary institution. J. Gastrointestin. Liver Dis. 19: 69-73.

Genetics and Molecular Research 13 (4): 9271-9284 (2014)

CFUNPEC-RP www.funpecrp.com.br 
Kim SH, Kim HJ and Lee JI (2008). Multiple primary cancers including colorectal cancer. J. Korean Soc. Coloproctol. 24: 467-472.

Liu F, Qin D and Wang Q (1979). Clinical pathological analysis of 172 cases with multiple primary carcinoma. Chin. J. Oncol. 1: 113-119.

Morgenfeld EL, Tognelli F, Gil Deza E, Santillan D, et al. (2003). Synchronous and metachronous second (ST) and third (TT) primary tumors (PT) in a large patient population. Proc. ASCO 22: 3152.

Noronha V, Berliner N, Ballen KK, Lacy J, et al. (2006). Treatment-related myelodysplasia/AML in a patient with a history of breast cancer and an oligodendroglioma treated with temozolomide: case study and review of the literature. Neuro. Oncol. 8: 280-283.

Oya M, Takahashi S, Okuyama T, Yamaguchi M, et al. (2003). Synchronous colorectal carcinoma: clinico-pathological features and prognosis. Jpn. J. Clin. Oncol. 33: 38-43.

Papajík T, Myslivecek M, Sedova Z, Buriankova E, et al. (2011). Synchronous second primary neoplasms detected by initial staging F-18 FDG PET/CT examination in patients with non-Hodgkin lymphoma. Clin. Nucl. Med. 36: 509512.

Rondeau V, Mathoulin-Pélissier S, Tanneau L, Sasco AJ, et al. (2010). Separate and combined analysis of successive dependent outcomes after breast-conservation surgery: recurrence, metastases, second cancer and death. $B M C$ Cancer 10: 697-709.

Wang XW, Liu L, Wang YW and Siegel R (2005). Clinical course of patients with small cell lung cancer as second primary malignancy. Chin.-Ger. J. Clin. Oncol. 4: 297-300.

Warren S and Gates O (1932). Multiple primary malignant tumors. A survey of the literature and statistical study. Am. $J$. Cancer 16: 1358.

Yamamoto S, Yoshimura K, Ri S, Fujita S, et al. (2006). The risk of multiple primary malignancies with colorectal carcinoma. Dis. Colon. Rectum 49: S30-S36.

Zhu LF, Xue P and Wang LW (2010). Retrospective investigation of 65 cases with multiple primary cancers. Fudan Univ. J. Med. Sci. 37: 591-593. 